\title{
A de novo nonsense mutation in the tyrosine kinase lyn in a patient with an early onset autoinflammatory phenotype
}

\author{
Adriana Almeida De Jesus ${ }^{1}$, Gina Montealegre ${ }^{1 *}$, Yin Liu1, Bernadette Marrero', Hyesun Kuehn², Katherine Calvo², \\ Sergio Rosenzweig ${ }^{2}$, Thomas Fleisher ${ }^{2}$, Richard Chyi-Chia Lee ${ }^{3}$, April Brundidge ${ }^{1}$, Dawn Chapelle ${ }^{1}$, Yan Huang ${ }^{1}$, \\ Stephen Brooks', Susan Moir ${ }^{4}$, Eric Meffre ${ }^{5}$, Melinda Merchant ${ }^{3}$, Zuoming Deng ${ }^{1}$, Raphaela Goldbach-Mansky ${ }^{1}$
}

From 21st European Pediatric Rheumatology (PReS) Congress

Belgrade, Serbia. 17-21 September 2014

\section{Introduction}

Lyn kinase is a Src-family tyrosine kinase expressed by hematopoietic and non-hematopoietic cell types. It functions as an activactor and an inhibitor of signaling pathways. Phosphorylation of a tyrosine residue at position 508 keeps the molecule in an inactive form. Lyn $^{\text {up/up }}$ mice have a gain-of-function mutation generated at the tyrosine position 508 (Y508F) and present with severe anemia, autoimmune glomerulonephritis and positive ANA. Using whole exome sequencing (WES), we identified a pediatric patient with a nonsense de novo mutation in lyn kinase presenting with an early-onset autoinflammatory phenotype.

\section{Objectives}

To describe the successful characterization by WES of a patient with a de novo mutation in LYN, a gene not previously associated with human disease.

\section{Methods}

Genomic DNA was extracted from total blood of the patient and his parents and WES was performed using human exome capture Agilent V4 enrichment kit and Illumina HiSeq2000 platform.

\section{Results}

A 3 year-old Caucasian male born at 31 weeks' gestation presented with hydrops fetalis, requiring intrautero blood transfusion. Post partum he developed

${ }^{1}$ National Institute of Arthritis Musculoskeletal and Skin diseases (NIAMS), Bethesda, USA

Full list of author information is available at the end of the article hepatosplenomegaly, purpuric skin rash, periorbital erythema and testicular swelling. Laboratory exams showed increased $C$-reactive protein (CRP) anemia, severe thrombocytopenia and increased liver enzymes. At 9 months of age a splenectomy was performed for refractory thrombocytopenia. Post splenectomy, he developed leukocytosis, thrombocytosis and anemia persisted. A liver biopsy showed periportal lymphocytic infiltrate and vanishing bile duct disease. He had vasculitis on a skin biopsy and circulating autoantibodies (positive ANA, anti-Sm, anti-SSA, anti-phospholipids and anti-mitochondrial antibodies). Despite some improvement after initiation of prednisone and IVIG infusions, prednisone doses could not be weaned. We identified a de novo mutation in a candidate gene $(L Y N)$ by WES. The mutation was confirmed as de novo by Sanger sequencing. The change from cytosine to guanine $(\mathrm{c} .1524 \mathrm{C}>\mathrm{G})$ leads to a premature stop-codon at the tyrosine position (Y508*) with loss of the phosphorylation site. B lymphocytes showed a constitutive phosphorylation of lyn and B cell phenotyping showed a significantly diminished frequency of immature B cell populations in peripheral blood and in bone marrow. $B$ cell activation was reduced upon IgM stimulation in comparison with healthy controls. The genetic defect identified in this patient led us to initiate treatment with a tyrosine kinase inhibitor (dasatinib). After 6 months of treatment, clinical improvement was observed with lower frequency of disease flares, resolution of anemia, improvement of leukocytosis, thrombocytosis and liver enzymes. A decrease of prednisone dose to physiologic levels and discontinuation of IVIG infusions were also achieved. 


\section{Conclusion}

A lyn kinase nonsense mutation was detected in a patient with a severe early-onset autoinflammatory phenotype. Treatment with dasatinib was shown to be promising in the patient described.

\section{Disclosure of interest}

None declared.

\section{Authors' details}

'National Institute of Arthritis Musculoskeletal and Skin diseases (NIAMS), Bethesda, USA. ${ }^{2}$ Department of Laboratory Medicine, Bethesda, USA.

${ }^{3}$ National Cancer Institute, Bethesda, USA. ${ }^{4}$ National Institute of Allergy and Infectious Diseases, National Institutes of Health, Bethesda, USA.

${ }^{5}$ Yale University, New Haven, USA.

Published: 17 September 2014

- Convenient online submission

- Thorough peer review

- No space constraints or color figure charges

- Immediate publication on acceptance

- Inclusion in PubMed, CAS, Scopus and Google Scholar

- Research which is freely available for redistribution 